\title{
Leadership, Artificial Intelligence and the Need to Redefine Future Skills Development
}

\author{
Lize Moldenhauer \\ OMNI Academy for Education, Training and Development, South Africa \\ Cindy Londt \\ OMNI Academy for Education, Training and Development, South Africa
}

The evolution of artificial intelligence has resulted in the need to establish alignment of artificial intelligence to various avenues of life and that of Leadership in an Artificial Intelligence based economy. Challenges associated to the independence of artificial intelligence, in addition to possible autonomy, are the focus of thought. As part of this paper, the focus is on the links between artificial intelligence and leadership, and its relationship, if any. A case study has been done on an organisation that has developed a model for the training of staff towards the AI model of leadership.

\section{INTRODUCTION}

Leadership and Artificial Intelligence (AI) are directly related, as it is through the actions of leaders that $\mathrm{AI}$ is funded, designed and created. There is a tendency to consider the negative aspects of AI, as noted in McDermott (2007), where the challenges of the unknown are marginalised. Rather than to challenge the efficacy and potential of AI, this paper considers that learning and development are key to ensuring that future leaders are developed to understand AI, develop strategic objectives which include the interrelated functions of technology, knowledge management and the AI that it initiates. In addition to this, leaders are required to develop opportunities that do not create challenges between humans and AI in the form of work, or the loss of potential work by the human form. The focus on qualifications, linked specifically to the term leadership, have been developed like a Master's in Business Leadership, at the University of South Africa, to help differentiate the focus on business administration to the focus on skills required of leaders. The evolution of artificial intelligence has resulted in the need to establish alignment of artificial intelligence to various avenues of life and that of Leadership in an Artificial Intelligence based economy. Training Leaders therefore is primary, and a curriculum should be developed that provides the tools to ensure the ongoing efficacy of AI engagement. The potential curriculum and programme is explored for Leaders in such a way as to engage in the positive based future of AI.

\section{LITERATURE REVIEW}

This literature review considers AI and its relationship to Leadership. This does not imply that one is isolated from the other, but through the initial discussion on $\mathrm{AI}$ and its evolution, the focus of $\mathrm{AI}$ and its 
current and future intention is understood as both independent and co-dependent. This is contrasted by Knowledge Management, which indicates that there is a potential power-based relationship between Leaders and AI based on the intention of the Leader. Finally, a partnership between AI and Leaders is explored to understand that there is a potential for mutually beneficial situations if the value of AI is understood and the purpose of Leaders explored.

\section{HISTORICAL RESEARCH AI}

Research from the 80s and 90s considers the role of AI and notes that technology is rapidly evolving and provides ongoing critical reflection. Newell (1982) evaluated research on AI from 1955 to 1980 and noted that AI had evolved not in a stereotypical manner, but had been linked to accumulation and assimilation. In addition, Newell notes that AI has not provided the opportunity for providing the scientific answers that are expected of it. Mataric (1993, pg. 40) notes, "[s]ynthesizing and observing group behaviour will offer insights into understanding social interactions in biology, as well as help derive methods for principles behaviour control on robotics and AI." Through considering insights and exploring behaviour, results have shown that AI does indeed have opportunities for providing services in the future that are revolutionary in what has previously been considered servitude based roles, such as housekeeping, basic administration and bookkeeping services.

The development of research like, The International Dictionary of Artificial Intelligence (Raynor, 1999), has helped to define the behaviour, service and principles of AI. The evolution of AI has changed the perception of AI as servitude based, to one in which it is perceived as interactive. Winograd (1997, pg. 8) notes that $\mathrm{AI}$, and "[s]uccessful interaction design requires a shift from seeing the machinery to seeing the lives of the people using it. In this human dimension, the relevant factors become hard to quantify, hard to even identify."

From interaction design, the focus has been on human interaction and AI interaction, to ensure efficacy of the system. As Moursund (2006, pg. 27) notes:

AI programs make use of both algorithmic and heuristic procedures. Many of the problems being addressed using AI have the two characteristics:

1. The problems are very difficult from a human point of view, and a human worldclass expert does not solve them perfectly. Indeed, many of the problems are beyond human (unaided by computers) capabilities.

2. The computer programs being written to address these problems make use of algorithms, heuristics, computer speed, computer storage capacity, and computer connectivity. This approach can produce ICT systems of increasing expertise within many different problem-solving areas of interest to people but cannot guarantee success in solving all of the problems within each of these areas.

Historically, AI has evolved and improved and thus become more functional. The evolution has resulted in moving from the conceptual ideas of the 80 s to practical models provided in the $2000 \mathrm{~s}$. In part, Savarimuthu et al. (2007), notes that the evolution of AI has resulted in the consideration of developing the practical models and in so doing provides cognitive functions that are working to contribute to primary service functions. As Yudkowsky, (2008, pg. 17) states, "AI can rewrite its existing cognitive functions to work even better".

However, not all of the historic studies speak positively of AI. Education has had access to computers from the 80's as explored by Schofield et al. (1989), despite this, it has not resulted in teachers and students using the computers due to a of lack of understanding of how to use the technology to address the challenges of educators. Similarly, there are instances were researchers like McDermott (2007) and Brunette et. al (2009), consider consciousness studies of AI and the lack of researchers desires to address the contentious and controversial issues thereof. This results in proposed models, but without actual implementation. 


\section{Knowledge Management}

Knowledge is critical to the understanding of AI, as knowledge management has various functions which have been understood and documented in relation to how AI is developed. Leadership is the key to the distribution of knowledge. As Liebowitz (2001, pg. 4) notes, "[k]nowledge distribution, one of the basic functions of knowledge management, involves sending knowledge internally and externally to those who could benefit from the use and application of the knowledge." Leaders understand this function and whether positive or negative, influence how knowledge distribution happens. Leadership and AI are considered to be power focused due to the nature of sharing information and knowledge distribution. This can be understood from an agent versus leader perspective, as AI focuses on a specific stream of engagement, namely for the "leader" that provides it with its identity and information. This is well defined by Bastin et. al (2007, pg. 11),

There is no explicit notion of leadership as each agent maintains an internal model of who its leader is. In our work, each agent chooses its leader explicitly and the leader sends the norms only to its followers. Also, the agents in our model have the notion of autonomy which is more representative of a realistic society.

The fact that leaders send information to followers ensures that a power relationship and control exists which in turn ensures the pursuit of power. Van Harmelen et al. (2008) note that even though there is a sense that the AI functionality will be human-like, there is a belief that power can be the driving force behind it. Leadership with positive goals will ensure a positive experience while those with negative or self-interest related goals will have alternative means. Dewhurst and Wilmott (2014, pg. 5) ask the question: "If senior leaders successfully fuel the insights of increasingly brilliant machines and devolve decision-making authority up and down the line, what will be left for top management to do?" They address the question and note that the role for the individuals with the strongest judgments are the senior leaders.

This field of leadership is fraught with difficulties in that it is a "new world of analytical complexities." As such, experiments need to be conducted with the intention of discovering changes which consider the ramifications of the new insights, rules and managerial styles. AI has the ability to address the issues by informing leaders of such issues.

Articulation, the form of attaching sensors and understanding the functions through digitization, also determines how knowledge management is progressed (Herschel \& Jones, 2005). This is still linked to how information is shared and how the information has the opportunity to control.

One of the critical requirements of leaders in AI related functions is to ensure that there is a knowledge management system which is inclusive but has clear systems and initiatives. Liebowitz (2001, pg. 3) notes that:

The major lesson learned is that most organizations do not have a rigorous, comprehensive knowledge management methodology for creating these knowledge management systems and initiatives. Part of the reason for this phenomenon is that the knowledge management providers themselves do not have comprehensive knowledge management methodologies.

Thus, even though AI is developed, linked to knowledge management systems and implemented through simulation and other activities, integrated systems and comprehensive knowledge management methodologies still require enhancement to enable articulation to Leader paradigms. If understood so well against a singular leadership goal then as Harmelen, Lifschitz \& Porter (2008) note, "computers can be made to exhibit human-like abilities" (pg. vii), even if this is negative. Coupled with this is anonymity, and the fact that identity can be created and managed accordingly, without representing an authentic original identity (Asenbaum, 2017). 


\section{Partnering with Automation}

Automation and the development of AI provides opportunities for a change in job related function from mundane to innovative work in technical skills and engineering. The advent of AI has implications for the labour force. At its current level, AI threatens to replace certain fields of expertise within an organisation which may result in labour disputes. As such, management needs to address these issues by implementing training strategies for new fields of advancement, taking into account the need for human job satisfaction as well as the need to implement roles for AI. With AI comes the ability to link learning to it and more specifically personalized learning experiences. Polson et al. (2010, pg. 186) note, "[i]f effectively exploited for teaching and learning, intelligent systems can offer unique opportunities to deliver personalized learning experiences and to form reciprocal relationships between formal and informal learning groups and environments." This is further supported by research conducted by the McKinsey Global Institute (2017), which recognises the reduction in labour costs. The efficacy of throughput which can be met ensures that reliability is in place as well as the maintenance of quality. These are all win-win situations, which ensure that the products produced are of better quality, and that opportunities for other roles come into place as potential employment opportunities.

The use of predictive modelling has the potential to change years of research and inferencing (Bennett. et al., 2012) and provide actual products which are specific to ongoing recommendations in the health care industry. Although this does not challenge decades of research, it does however provide opportunities for quicker resolution in empowering the health care industry in providing better services.

Regardless, there are some challenges which partnering with automation brings. These challenges do not have to be the barrier to the success of Leaders initiating efficiency, but rather provide regulation and recommendations of best practice models. Scherer notes, (2016, pg. 400):

We are entering an era where we will rely upon autonomous and learning machines to perform an ever-increasing variety of tasks. At some point, the legal system will have to decide what to do when those machines cause harm and whether direct regulation would be a desirable way to reduce such harm. This suggests that we should examine the benefits and drawbacks of AI regulation sooner rather than later.

The key to successful Leadership is the engagement with the drawbacks in ensuring the success of automation in conjunction with its goals and desires. The benefits of using AI seem to provide more opportunities than challenges.

\section{RESEARCH METHODOLOGY}

The purpose of the research is exploratory: to understand potential models for engaging with learning and development-initiated projects for Leadership and AI efficacy. The research methodology used was qualitative in nature. Data was not based on statistics but rather engagement with linking curriculum to leadership development initiatives.

The initial phase of the research included a literature review to understand the AI, its function, the relationship between AI and Knowledge Management and more importantly partnership available with AI and Leadership. Findings from this research were then considered for understanding the potential learning programmes that could be developed to help develop Leaders within the AI innovation framework.

"Descriptive Case Studies" are noted as being controversial (McDonough, 1997; Zainal, 2007), as they are considered subjective. This research intends to engage with stakeholders on a potential training initiative and therefore the recommendations of the training programme are exploratory, and the curriculum is at the initial design phase.

Focus groups were conducted with the operational managers as well as the executive managers who are based in the private educational sector of South Africa: one to gather the initial understanding of the models and the intention of Leadership and AI and the second with a view towards the practical implementation of such a learning programme. 
This is not a comprehensive evaluation of multiple stakeholders but rather a singular case and the application thereof.

\section{Developing a Learning Programme for Leaders Aligning to Artificial Intelligence}

Taking into account the information identified, in order to best partner with AI, Leaders need to ensure that human capital management learning programmes are developed to consider that functional roles implemented by $\mathrm{AI}$ are accompanied by redefined:

1. Competency Management

2. Workforce Planning

3. Organisation Design

4. Talent Analytics

Competency Frameworks have to consider AI and the role it has in replacing staff, functions and overall business output. The learning programme has to encourage that organisational efficacy is not only limited to staff but also linked to the technology (AI) that accompanies it, and the potential development of AI which can replace certain roles.

In addition to this, to ensure business goals are met, the following are considered:

- Reward Management

- Performance Management

- Capability Management

- Career Management.

Full skills audits should be conducted to establish knowledge, skills and aptitudes, and the organisation should include psychometric assessment that leads to the highlighting of the current challenges within the leadership mix/composition of the organisation.

Resilience and Analytical/Logical reasoning are highlighted as low measured aptitudes. It would be suggested that a detailed skills audit is conducted to ensure all critical areas of development are evaluated so that the Learning and Development Strategy of the organisation can be formulated to support the business growth targets. The risk if an integrated plan is not formalised is that Learning, and Development will approach business needs with a reactive agenda. In addition, this will allow for an integrated brand alignment to the conceptual thought process of creating a learning function which will also support effective succession planning and development strategies of the organisation.

In addition to these, the following should be developed to help align the minds of Leaders to AI:

- Complex Problem Solving (complicated or intricate, hard to understand or deal with)

- Critical \& Computational Thinking (managing massive amounts of data)

- Creativity (original ideas for creating out of the box solutions / blue sky thinking)

- People Management (management, leadership and influence)

- Collaborative Management (cross functional and complex initiatives)

- Emotional Intelligence (self-awareness \& regulation, motivation, empathy and social skills)

- Judgment and Decision Making (thinking, cognitive process or act of reaching a decision, drawing sound conclusions, forming an opinion through assessment, comparison, and/or deliberation)

- Service Orientation (ability and desire to anticipate, recognise and meet others' needs before it is articulated)

- Negotiation Skills (priority classification and compromise discussions for results)

- Cognitive Flexibility (adapt strategies to face new and unexpected conditions in the environment)

Overall, the focus of the programme is the engagement with future Leaders to understand the changing world, how the focus is linked to technology and data and the key requirements thereof. 


\section{RECOMMENDATIONS}

As noted in section 4, it is recommended that AI based learning programmes are developed; ones which specifically look at Leadership to ensure that there is sufficient engagement at a senior level.

In addition to this, Knowledge Management Systems need to be evaluated for efficacy, and the role of $\mathrm{AI}$ and Knowledge Management explored as directly linked to the organisation. This then becomes a key driver in the development of learning programmes which share the Knowledge Management Systems and the impact that AI has on them.

Finally, partnering with automation and more specifically AI efficacy within an organisation, to ensure compliance with regulation, legislation, staff and potential challenges is critical in ensuring Leadership in 2030 is current.

\section{CONCLUSION}

AI has its challenges and Leaders need to provide the correct engagement with it, and with those that work with them, to ensure that there is coherence between all. As is the potential with many new applications of technology within businesses, there are risks as well as opportunities. The failure to adopt new technologies, as is often the case, leads to failures of the business itself. As such, it is taken as a given that new technologies must be adopted and experimented with in order to gain success in a competitive environment.

There are two options: One, in which AI is repelled, ignored, not developed nor implemented due to design not finding the opportunities for development. This indicates a negative link to AI, but also provides the opportunities for control over AI;

and secondly, to engage with Leaders to ensure that technology is embraced, that systems which can change the quality of lives are explored, to encourage the development of technology and research and ensure that staff look at technology as the source of improvement with fundamental new opportunities.

It is through learning that Leaders as well as potential Leaders have the opportunity to provide the engagement with how technology has the power to change efficiency, provide services, improve the quality of life and develop operational practices that always provide optimal service. Therefore, it is suggested that hybridising the workforce with AI is necessary as it will improve workplace culture for both employees and leaders.

\section{REFERENCES}

Asenbaum, H. (2017). Cyborg Activism: Exploring the reconfigurations of democratic subjectivity in Anonymous. New Media \& Society, 1-21. Retrieved from http://journals.sagepub.com/doi/full/10.1177/1461444817699994

Bennett, C.C., Doub T.W., \& Selove, R. (2012). EHRs Connect Research and Practice: Where Predictive Modeling, Artificial Intelligence, and Clinical Decision Support Intersect. Health Policy and Technology. In Press.

Brunette, E. S., Flemmer, R. C., \& Flemmer, C. L. (2009). A Review of Artificial Intelligence. Proceedings of the 4th International Conference on Autonomous Robots and Agents. Wellington, New Zealand, pp 385-392.

Dewhurst, M., \& Willmott, P. (2014, September). Manager and machine: The new leadership equation. McKinsey Quarterly, 1 - 8. Retrieved from $\mathrm{http}: / /$ www.mckinsey.com/insights/leading_in_the_21st_century/manager_and_machine?cid $=\mathrm{mc}$ kq50-eml-alt-mkq-mck-oth-1409 acesso 16 set 2014

Herschel, R. T., \& Jones, N. E. (2005). Knowledge management and business intelligence: the importance of integration. Journal of Knowledge Management, 9(4), 45-55. Emerald Publishing Group.

Liebowitz, J. (2001). Knowledge management and its link to Artificial Intelligence. Expert Systems with Applications. Pergamon. Elsevier Science Ltd. Volume 20 (2001). pp 1-6. 
Mataric, M. J. (1993). Designing Emergent Behaviors: From Local Interactions to Collective Intelligence. In Meyer, J-A., Roitblat, H. and Wilson, S. (eds.). Proceedings, Simulation of Adaptive Behavior. MIT Press. pp 432-441

McDermott, D. (2007). Artificial Intelligence and Consciousness. Chapter 6 of Zelazo, P. D., Moscovitch, M. and Thompson, E (eds.), Cambridge Handbook of Consciousness. Cambridge University Press. pp 117-150.

McKinsey \& Company. (2017). McKinsey Global Institute, A Future that Works: Automation, Employment, and Productivity, January 2017 Executive Summary. McKinsey \& Company.

Moursund, D. (2006). Brief Introduction to Educational Implications of Artificial Intelligence. University of Oregon.

Newell, A. (1982). Intellectual Issues in the History of Artificial Intelligence. Appears in Machlup, F. and Mansfield U. (eds.) The Study of Information: Interdisciplinary Messages. John Wiley and Sons. New York.

Polson, D. and Morgan, C. (2010). Towards an intelligent learning system for the natural born cyborg. Journal of the Research Center for Educational Technology, 6(1). pp. 185-193.

Raynor, W. (1999). The International Dictionary of Artificial Intelligence. The Glenlake Publishing Company, Ltd. United States of America.

Savarimuthu, B. T. R., Cranefield, S., Purvis, M. and Purvis, M. (2007). Role Model Based Mechanism For Norm Emergence In Artificial Agent Societies. The Information Science Discussion Papers. University of Otago. No. 2007 (2).

Scherer, M. (2016). Regulating Artificial Intelligence Systems: Risks, Challenges, Competencies, and Strategies. Harvard Journal of Law \& Technology, Volume 29, Number 2 Spring 2016.

Schofield, J. W., Evans-Rhodes, D. and Huber, B. R. (1989). Artificial Intelligence in the Classroom: The Impact of a Computer-Based Tutor on Teachers and Students. Research was sponsored by the Cognitive Science Program, Cognitive and Neural Sciences Division, Office of Naval Research. United States.

Van Harmelen, F., Lifschitz, V. and Porter, B. (eds.). (2008). Handbook of Knowledge Representation. Elsevier, Oxford, United Kingdom.

Winograd, T. (1997). From Computing Machinery to Interaction Design. Published online by permission of Denning, P. and Metcalfe, R. (eds.), Beyond Calculation: The Next Fifty Years of Computing. Springer-Verlag. pp 149-162.

Yudkowsky, E. (2008). Artificial Intelligence as a Positive and Negative Factor in Global Risk. Global Catastrophic Risks. Bostrom, N. and Ćirković, M. M. (eds.). Oxford University Press, New York. pp 308-345. 